\title{
Energy-Balanced Transmission Policies for Wireless Sensor Networks
}

\author{
A.K.M. Azad, Student Member, IEEE, and Joarder Kamruzzaman, Member, IEEE
}

\begin{abstract}
Transmission policy, in addition to topology control, routing, and MAC protocols, can play a vital role in extending network lifetime. Existing transmission policies, however, cause an extremely unbalanced energy usage that contributes to early demise of some sensors reducing overall network's lifetime drastically. Considering cocentric rings around the sink, we decompose the transmission distance of traditional multihop scheme into two parts: ring thickness and hop size, analyze the traffic and energy usage distribution among sensors and determine how energy usage varies and critical ring shifts with hop size. Based on above observations, we propose a transmission scheme and determine the optimal ring thickness and hop size by formulating network lifetime as an optimization problem. Numerical results show substantial improvements in terms of network lifetime and energy usage distribution over existing policies. Two other variations of this policy are also presented by redefining the optimization problem considering: 1) concomitant hop size variation by sensors over lifetime along with optimal duty cycles, and 2) a distinct set of hop sizes for sensors in each ring. Both variations bring increasingly uniform energy usage with lower critical energy and further improves lifetime. A heuristic for distributed implementation of each policy is also presented.
\end{abstract}

Index Terms-Wireless sensor networks, energy efficiency, optimal transmission range.

\section{INTRODUCTION}

$\mathrm{R}_{\mathrm{h}}^{\mathrm{E}}$ ECENT developments in integrated circuit technology have made possible the construction of tiny and lowcost sensor nodes with on board sensing, signal processing, and wireless communication capabilities. A wireless sensor network (WSN) is a collection of such sensor nodes spatially deployed in an ad hoc fashion that performs distributed sensing tasks in a collaborative manner without relying on any underlying infrastructure support [1], [2]. The envisaged flexibility and ease of deployment are some of the reasons for its numerous applications [3], [4], [5], [6], such as, environment monitoring, surveillance systems, target tracking, health care systems, emergency navigation, traffic management, etc.

Unlike traditional wireless networks, WSNs are different in communication paradigm. For example, communication is any-to-any in wireless ad hoc networks while in WSNs all nodes send their data toward a common sink. This manyto-one communication paradigm results in a highly nonuniform energy usage among sensors located at varying distances from the sink. Moreover, sensor nodes have limited and, in many cases, irreplaceable or irrechargable power sources, hence efficient usage of energy is the key for longer network lifetime and sustained coverage of deployed area. To address this issue, most studies in WSNs [7], [8], [9], [10], [11], [12] have concentrated on the design of energy efficient routing protocols, medium access control protocols, clustering techniques, and topology control

- The authors are with the Faculty of Information Technology, Gippsland School of Information Technology, Monash University, Churchill, VIC 3842, Australia. E-mail: \{akm.azad, joarder.kamruzzaman\}@monash.edu.

Manuscript received 24 Oct. 2008; revised 18 May 2009; accepted 19 Jan. 2010; published online 16 Dec. 2010.

For information on obtaining reprints of this article, please send e-mail to: tmc@computer.org, and reference IEEECS Log Number TMC-2008-10-0426. Digital Object Identifier no. 10.1109/TMC.2010.238. mechanisms. Recent studies show that, in addition to these techniques, choosing appropriate transmission policy [13], [14], [15], [16] for transporting sensor data to the designated sink plays a crucial role in limiting lifetime as nodes spend most of their energy for radio transmission and reception. However, much attention has not been given in this area. With the aim to improve overall network lifetime, a transmission policy should have following features:

1. Multihop: Transmission power increases exponentially by the $\gamma$ th power of distance, where $2 \leq \gamma \leq 4$ is the path loss factor. Single-hop transmission of data to the sink causes rapid depletion of energy for long range applications. Thus, an energy efficient transmission policy is necessarily multihoping.

2. Variable transmission range: Multihop transmission with fixed transmission range creates hot spots since a small number of nodes near the sink need to relay all the incoming traffic from the outer nodes, hence die out quickly reducing the network's lifetime drastically even though many nodes still have considerable amount of residual energy. Varying transmission range over time attains more uniform traffic and energy usage distribution among sensors.

3. Energy balanced duty cycles: The number of cycles nodes use a particular transmission range is crucial to achieve balanced energy usage among sensors. Thus, a transmission policy should optimally determine duty cycles for each transmission range with the objective to maximize overall network lifetime.

4. Regularity: Scheduling sensors avoiding interference needs a great deal of effort [17]. The transmission ranges and associated duty cycles to be used by sensors should maintain certain pattern so that a set of noninterfering nodes can be scheduled together. 
Bhardwaj et al. [13] studied the upper bound on the lifetime of a sensor network by finding an optimal hop distance to send a packet from sensor to the sink to minimize total energy usage along the path. The authors considered uniform relay traffic on the intermediate nodes along the multihop path. But this study is of limited use since in WSNs, relay traffic is higher on nodes closer to the sink than nodes near the boundary. Mhatre and Rosenberg [14] studied single-hop ( $\mathrm{SH}$ ) and multihop ( $\mathrm{MH})$ transmissions and obtained an optimal transmission distance for $\mathrm{MH}$ communication. A hybrid of $\mathrm{SH}$ and $\mathrm{MH}$ transmission was also proposed and the optimal ratio of the number of data cycles operated in these two modes over the network lifetime was obtained. Efthymiou et al. [15] also proposed a hybrid of SH and $\mathrm{MH}$ transmission to attain balanced energy usage among sensor nodes. Both in [14] and [15], the authors divided the network into cocentric circular rings. In [14], all sensors follow the same ratio in the number of $\mathrm{SH}$ and $\mathrm{MH}$ transmissions, while the ratio is ring wise different in [15].

While ring-based approach together with the optimal transmission distance seems attractive, fixed transmission distance and sending data only one ring forward in $\mathrm{MH}$ transmission contribute to the collapse of the innermost ring and result in highly nonuniform energy drainage which ultimately reduces lifetime. With the aim to maximize network lifetime and achieve better energy usage distribution among sensors, this paper makes the following contributions. First, assuming cocentric rings around the sink, we express transmission distance in terms of ring thickness and hop size (number of rings a sensor forward its traffic toward the sink during each transmission) and obtain their optimal values to maximize network lifetime. Second, detail mathematical analysis of traffic distribution over the rings is presented as a function of ring thickness and hop size. Third, energy usage distribution and how the critical energy changes with hop size variation are analyzed. Finally, on the basis of the above findings, we propose three transmission policies, namely, fixed hop size (FHS), synchronous variable hop size (SVHS), and asynchronous variable hop size (AVHS) transmissions. These transmission policies differ in terms of their degree of flexibility in using variable transmission ranges and their associated duty cycles among sensor nodes. Moreover, we presented distributed heuristics for SVHS and AVHS transmissions, namely, heuristic-SVHS (H-SVHS) and heuristic-AVHS (H-AVHS), respectively, by exploiting the inherent energy usage distribution pattern among sensors for varying hop sizes. Performance analysis shows substantial improvement of network lifetime over the existing $\mathrm{SH}, \mathrm{MH}$, and their hybrid transmission policies irrespective of network parameters. Energy usage is more uniformly distributed over the rings and critical energy per data cycle is reduced significantly in our policies.

The rest of this paper is organized as follows: In Section 2, we discuss the system models and formally define transmission policy as a problem. We review $\mathrm{SH}, \mathrm{MH}$, and their hybrid transmissions in Section 3. In Section 4, we propose our transmission policies: FHS, SVHS, and AVHS transmissions together with vigorous analytical formulation. Section 5 presents the distributed heuristics for SVHS

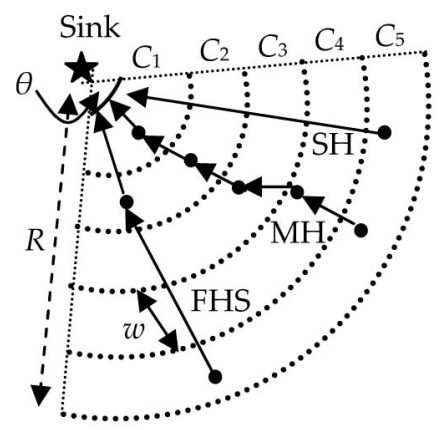

Fig. 1. Single hop, multihop, and fixed hop size with hop size, $\eta=3$ transmissions.

and AVHS transmissions. The performance of the proposed policies has been illustrated in Section 6, and Section 7 concludes the paper.

\section{The Models and Problem Statement}

We consider sensors are randomly and uniformly deployed in a region where each sensor measures the value of some spatial function (say, temperature and/or air quality) and the sensed data are sent to the sink. We are interested in obtaining energy efficient transmission policy so that the overall network lifetime is maximized.

\subsection{Network Model}

We consider a WSN where a number of sensors are randomly and uniformly deployed over the network area. Let $E_{\text {init }}$ be the initial energy storage in a sensor node. We formulate our transmission policies for a circle sector having radius $R$ and angle $\theta$ at the sink as shown in Fig. 1 and consists of $n$ sensors. The motivation behind such consideration is that, any polygonal-shaped network area could be approximated by such circle sectors [15]. Moreover, in clustered network, a cluster can be approximated by one or more circle sectors [14], [16]. We divide the whole sensor field into a set of $l(=R / w)$ cocentric circular ring areas having ring thickness of $w$, as shown in Fig. 1. A sensor " $s$ " having distance $d_{s}$ from the sink is located in ring $C_{i}$, if and only if $(i-1) w<d_{s} \leq i w$. Since the number of sensors in a certain region is proportional to the size of the area, the number of sensors located in ring $C_{i}$, denoted by $N(i, w)$, and the number of sensors located in rings $C_{i+1}$ to $C_{l}$, i.e., at the outer rings relative to ring $C_{i}$, denoted by $\tilde{N}(i, w)$, are given by (1) and (2), respectively,

$$
\begin{aligned}
& N(i, w)=(2 i-1) n w^{2} / R^{2}, \\
& \tilde{N}(i, w)=\left(R^{2}-i^{2} w^{2}\right) n / R^{2} .
\end{aligned}
$$

Sensors measure parameters like temperature, air quality or any physical events at the surrounding environment and data generated thereby are sent toward the sink. The amount of data generated depends on the event detected and we assume that $p_{k}$ is the probability that a sensor senses $k$ th event (among $m$ events) within its vicinity during a data cycle and once detected generates $\varsigma_{k}$ amount of data. During network lifetime, which is a considerably long period, events take place uniformly over the network area and the on average data generation by a sensor per data cycle can be estimated as 


$$
\lambda_{s}=\sum_{k=1}^{m} p_{k} \varsigma_{k}, \quad \sum p_{k}=1 .
$$

We decompose the transmission distance of traditional multihop communication into ring thickness and hop size. The hop size, denoted by $\eta \in\{1,2, \ldots, l\}$, is the the maximum number of rings a node forwards its data in a single transmission toward the sink. The transmission distance of a sensor in ring $C_{i}$, denoted by $x(i, w, \eta)$, is an integral multiple of ring thickness $w$ and will be determined by its ring index $i$ and hop size $\eta$.

\subsection{Energy Consumption Model}

The energy model specifies the energy consumption by sensor node during various operations such as radio transmission, reception, sensing, and computing. The energy spent for sensing and computing is relatively small, periodic, and same for all sensors in a particular WSN. Therefore, for simplicity, we adopt the energy model as in [14] considering the energy spent for radio transmission and reception that has direct impact on the choice of transmission policy. Let $\varphi_{t}$ and $\varphi_{r}$ be the amount of energy consumptions to transmit (to a distance $d$ ) and receive one bit of data, respectively, and are defined as

$$
\begin{aligned}
& \varphi_{t}=\alpha+\beta d^{\gamma}, \\
& \varphi_{r}=\alpha,
\end{aligned}
$$

where $2 \leq \gamma \leq 4$ is the path loss factor, $\alpha$ is the energy/bit needed to run the transceiver circuit, and $\beta$ is the energy consumed in amplifier circuit to transmit data.

\subsection{Network Connectivity}

Two important metrics for WSNs are connectivity and coverage. Deterministic sensor deployment may not often be feasible due to environmental constraint and thus network coverage and connectivity are probabilistic measures. For the nodes to successfully use multihop communication, it is necessary to ensure that at least the condition for node connectivity is met. Sufficient condition for node connectivity (with and without having coverage) considering random deployment of sensor nodes has been studied in [19]. For $n$ sensor nodes randomly deployed over a unit area, each having transmission range $r(n)$, the probability of node connectivity is given by [19, Lemma 3.1]

$$
\operatorname{Pr}\left(\text { Connectivity) } \geq 1-n e^{-\pi n r^{2}(n)} .\right.
$$

Normalizing the above relation for $n$ sensor nodes deployed over the sector having area $\frac{1}{2} \theta R^{2}$ instead of unit area and the desired probability for network connectivity being at least $p_{c o n}$, the minimum transmission range required by each sensor, denoted as $r_{c o n}(=r(n))$, is given by

$$
r_{c o n} \geq R\left[\frac{\theta}{2 n \pi} \log \frac{2 n \pi}{\theta\left(1-p_{c o n}\right)}\right]^{\frac{1}{2}} .
$$

\subsection{Problem Statement}

In mission critical applications, network lifetime is defined as the number of complete data cycles before the first sensor node runs out of energy [14], [16], [20]. Due to the energy constraint of individual sensor node, lifetime of a WSN is bounded by a finite number of data cycles. Ideally, we require that almost all the nodes in the network expire at about the same time. This ensures that very little residual energy is left when the system becomes unusable, i.e., when coverage/connectivity is lost. But achieving uniform distribution of energy usage among sensor nodes with improved network lifetime still remains a challenge. Sensors having the highest rate of energy usages per data cycle, denoted as critical nodes, limit the overall network lifetime. In this paper, we formulate transmission policies with the aim to achieve extended network lifetime through increasingly uniform distribution of energy usage among sensors.

In each transmission policy, we determine a vector $\Psi=$ $[\hat{w}, \hat{\mathbf{V}}]$ where $\hat{w}$ is the optimally determined ring thickness and $\hat{\mathbf{V}}=\{(\hat{\eta}, \hat{\partial})\}$ is the set of pairs consisting of optimal hop size $\hat{\eta}$ and respective duty cycle $\hat{\partial}$. Let $\xi_{s}(\hat{w}, \hat{\eta}, \hat{\partial})$ be the amount of energy usage by the sensor " $s$ " over $\hat{\partial}$ data cycles to send self-generated as well as relay traffic while using ring thickness $\hat{w}$ and hop size $\hat{\eta}$. Here and in subsequent formulations, the subscript " $s$ " refers to parameters related to a single sensor. Then the total energy consumed by the sensor under the transmission policy $\Psi$ is defined as

$$
\xi_{s}(\Psi)=\sum_{(\hat{\eta}, \hat{\partial}) \in \hat{\mathbf{V}}} \xi_{s}(\hat{w}, \hat{\eta}, \hat{\partial}) .
$$

The lifetime of the network under transmission policy $\Psi$, denoted by $L_{\Psi}$, is defined as the sum of duty cycles in $\hat{\mathbf{V}}$ so that $\xi_{s}(\Psi)$ is less than or equal to the initial energy $E_{\text {init }}$ for each sensor " $s$ ". The lifetime maximization thus can be formulated as the following optimization problem:

$$
\begin{aligned}
\operatorname{maximize} & L_{\Psi}=\sum_{(\hat{\eta}, \hat{\partial}) \in \hat{\mathbf{V}}} \hat{\partial} \\
\text { s.t. } & \quad \forall\left[\xi_{s}(\Psi) \leq E_{\text {init }}\right] .
\end{aligned}
$$

With same initial energy, the lifetime achievable for a WSN depends highly on the transmission policy $\Psi$.

\section{Transmission Policy Review}

In this section, we review three notable works found in the literature addressing transmission policy.

\subsection{Characteristic Distance for Transmission}

To find the optimal transmission distance at each hop that minimizes the total energy usage along the path, in [13], a data link between a radio transmitter and a receiver separated by $D$ meters are divided into $K$ subpaths by introducing $(K-1)$ intervening relay nodes. The authors have shown that, for given $D$ and the number of hops $K$, the overall energy dissipation along the path is minimum when length of all the subpaths are made equal to $D / K$ and the optimal number of hops is given by

$$
K_{\text {opt }}=\left\lfloor D / d_{\text {char }}\right\rfloor \text { or }\left\lceil D / d_{\text {char }}\right\rceil,
$$

where the distance $d_{\text {char }}$, called the characteristic distance, is independent of $D$ and is given by

$$
d_{\text {char }}=\left[\frac{2 \alpha}{\beta(\gamma-1)}\right]^{\frac{1}{\gamma}} .
$$

The above study assumes uniform relay traffic on the intermediate nodes along the multihop path which is not 
valid in WSN. The study focuses on one source-destination pair at a time without taking into account the many-to-one communication paradigm of WSNs where relay traffic is much higher on the nodes closer to the sink than that of the nodes near the network boundary.

\subsection{Energy Balanced Data Propagation}

Energy balanced data propagation in wireless sensor networks has been studied in [15] where the average per sensor energy dissipation during the entire lifetime is the same for all the sensors in the network. Once generated, a sensor sends data either one hop closer to the sink or directly to the sink.

In [15], the authors assume the network area as a cycle sector with the sink at the center. The cycle sector is divided into ring sectors or "slices" with each slice having thickness $r$. For balancing load and spreading energy dissipation evenly among sensors, a sensor in ring sector $T_{i}$ forwards data to $T_{i-1}$ (i.e., the next sector toward the sink) with probability $p_{i}$, while with probability $1-p_{i}$ it transmits data directly to the sink. There is a trade-off for choosing $p_{i}: 1$ ) if $p_{i}$ increases then transmissions tend to happen locally, thus energy consumption per transmission is low; however, sensors close to the sink tend to be overused since more data pass through them, and 2) on the other hand, if $p_{i}$ decreases, distant transmission by a sensor increases resulting in higher energy drainage by a sensor per transmission. The authors formulated a recursive relation to determine such probabilities for each ring sectors that guarantee energy balanced data propagation. Moreover, the authors also presented a close form estimate of $p_{i}$ [15, Theorem 5] considering energy consumption for transmission operation only. Similar approaches for the hybrid of $\mathrm{SH}$ and $\mathrm{MH}$ transmissions are also considered in [21] and [22], where the authors assume varying event detection rates and initial sensor energies for sensors in different ring sectors, and propose algorithms to find the probabilities of using $\mathrm{SH}$ and $\mathrm{MH}$ modes at different sectors considering path loss factor $\gamma=2$ and energy consumption for transmission only. In addition, in [22], the authors propose a "blind energy balanced data propagation" algorithm where the probability of the occurrence of events at different ring sectors is assumed not to be known a priori and is determined adaptively at the sensor nodes based on stochastic estimation methods.

Although the scheme attains balanced energy usage among sensors, use of either direct transmission to the sink or only one ring forward toward the sink results in overall energy inefficiency. Instead, better energy efficiency is expected when a combination of various hop sizes is used. Moreover, the authors do not mention about the optimal transmission range during multihop transmission. In the next section, we review $\mathrm{SH}, \mathrm{MH}$, and their hybrid transmission policies [14], where a close form optimal ratio among duty cycles for both communication modes as well as the optimal transmission distance during multihop transmission are presented considering both the transmission and reception energies.

\subsection{Single Hop, Multihop, and Hybrid Transmissions}

In [14], the authors analyzed the $\mathrm{SH}, \mathrm{MH}$, and their hybrid transmission policies. The authors assumed a heterogeneous
WSN divided into clusters and each cluster region is circular with the cluster head (sink) at the center. The whole cluster is divided into cocentric rings around the sink and sensors send their data toward the sink using single hop or multihop or combination of them.

In $\mathrm{SH}$ transmission, a sensor sends data directly to the sink (see Fig. 1). It is found that, in $\mathrm{SH}$ transmission, a significant amount of energy remains unused in sensors residing in rings $C_{1}$ to $C_{l-1}$ while sensors in the farthest ring $C_{l}$ is out of energy. A sensor in ring $C_{i}$ needs to send data to a distance of $i w$ on average to reach the sink directly, hence energy usages in each data cycle are

$$
\Omega_{s}(i, S H)=\left\{\alpha+\beta(i w)^{\gamma}\right\} \lambda_{s} .
$$

In $\mathrm{MH}$ communication in [14], data are relayed by a number of intermediate nodes on the way from the source node to the sink with one ring forward toward the sink at each hop (see Fig. 1). Here, nodes close to the sink (in $C_{1}$ ) need to relay all data coming from the sensors in the outer rings $\left(C_{2}\right.$ to $C_{l}$ ) and thus consume energy faster than sensors of any other rings, hence are the critical nodes. Using energy model as in Section 2.2, energy consumed by a sensor in ring $C_{i}$ in each data cycle is

$$
\Omega_{s}(i, M H)=\left(\alpha+\beta w^{\gamma}\right) \lambda_{s}+\left(2 \alpha+\beta w^{\gamma}\right) \frac{\tilde{N}(i, w)}{N(i, w)} \lambda_{s} .
$$

The authors find an optimal transmission distance $\hat{w}_{M H}$ (equal to the ring thickness) that minimizes the critical energy per data cycle, hence maximizes the network lifetime for $\mathrm{MH}$ transmission and is given by

$$
\hat{w}_{M H}=\left[\frac{4 \alpha}{\beta(\gamma-2)}\right]^{\frac{1}{\gamma}}, \quad \gamma>2 .
$$

As illustrated later in Fig. 5 (Section 6), in MH transmission energy dissipation rate among sensors decreases exponentially with their distance from the sink. As a result, in contrast to $\mathrm{SH}$ transmission, a significant amount of residual energy remains unused in sensors located at rings $C_{2}$ to $C_{l}$ while sensors in critical ring $C_{1}$ starts dying.

The authors also showed that, single hop is the better choice over multihop transmission for path loss factor $\gamma=2$ and vice versa for $\gamma=4$. Observing the two opposite energy decay characteristics a hybrid of single and multihop transmission policies was proposed in [14], and a unique ratio of the number of single hop, $n_{S H}$ and multihop, $n_{M H}$ data cycles as in (12) was obtained that would be followed by sensors in all rings.

$$
n_{S H}: n_{M H}=\Delta \Omega_{M H}: \Delta \Omega_{S H},
$$

where $\Delta \Omega_{M H}=\Omega_{s}(1, M H)-\Omega_{s}(l, M H)$ and

$$
\Delta \Omega_{S H}=\Omega_{s}(l, S H)-\Omega_{s}(1, S H) .
$$

Although hybrid transmission policy makes energy drainage in both ring $C_{1}$ and $C_{l}$ equal to the critical energy, energy usage by sensors still falls exponentially from ring $C_{2}$ (and from $C_{l-1}$ ) to the middle ring. This leaves substantial residual energy in $l-2$ number of rings out of total $l$ rings and thus the policy fails to achieve good energy usage distribution. Moreover, the network lifetime improvement is very insignificant (see Fig. 6). 


\section{Proposed Transmission Policies}

While multihop scheme seems attractive, using a fixed transmission distance and sending data only one ring forward as in [13], [14], [15], [16] contribute to the collapse of the innermost ring and result in highly nonuniform energy drainage. In the following, we present a transmission policy where data are forwarded by a hop size of multiple rings instead of one, and the optimum hop size and ring thickness are determined by formulating and optimizing the network lifetime as a function of hop size and ring thickness. Fig. 1 shows the data transmission for hop size 3 , where nodes in ring $C_{4}$ to $C_{l=5}$ transmit data three rings forward and nodes in ring $C_{1}$ to $C_{3}$ send data directly to the sink. Two other variations of this scheme are also presented by redefining the optimization problem that allows variation of hop sizes and respective duty cycles over lifetime. Detail mathematical analysis of traffic distribution and energy usage in each ring and network lifetime formulation in each scheme are presented below.

\subsection{Traffic Model}

In this section, we present an analytical model for the distribution of relay traffic among the sensor nodes. We assume the network model as described in Section 2.1. Let $I(i, w, \eta)$ be the cumulative incoming traffic in each data cycle on all $N(i, w)$ sensors in ring $C_{i}$ for given ring thickness $w$ and hop size $\eta$. The total incoming traffic on all sensors of ring $C_{i}$ is the sum of incoming traffic on sensors in ring $C_{i+\eta}$ and the total data generated by all sensors in ring $C_{i+\eta}$. Note that, data sent by sensors of ring $C_{l}$ are received by sensors in ring $\mathrm{Cl}-\eta$, hence, there is no incoming traffic on sensors located in rings $C_{l-\eta+1}$ to $C_{l}$. Thus, we can define $I(i, w, \eta)$ as the following recurrence relation:

$$
I(i, w, \eta)= \begin{cases}I(i+\eta, w, \eta)+N(i+\eta, w) \lambda_{s}, & 1 \leq i \leq l-\eta \\ 0, & \text { otherwise }\end{cases}
$$

The total incoming traffic would be distributed among $N(i, w)$ sensors in ring $C_{i}$. So the average incoming traffic on each individual sensor of ring $C_{i}$ per data cycle is

$$
I_{s}(i, w, \eta)=\frac{I(i, w, \eta)}{N(i, w)}
$$

Solution of the above recursive equation gives:

$$
I_{s}(i, w, \eta)= \begin{cases}\frac{l^{2}+\eta l-l}{n(2 i-1)} \lambda_{s}-\lambda_{s}, & 1 \leq i \leq \eta, \\ \frac{l^{2}-i^{2}+\eta l-i \eta-l+i}{\eta(2 i-1)} \lambda_{s}, & \eta<i \leq l-\eta, \\ 0, & i>l-\eta .\end{cases}
$$

From (14), it is trivial that, for a given ring thickness $w$ and hop size $\eta$, incoming traffic on a sensor decreases with increasing ring index $i$.

\subsection{Energy Consumption and Critical Ring}

Here we derive the energy consumption by a sensor for the traffic model proposed in Section 4.1. We also determine the critical ring, the ring with the highest rate of energy consumption per sensor per data cycle, and the critical energy. A sensor in ring $C_{i}$ transmits data $\eta$ rings forward toward the sink if $i \geq \eta$ and $i$ rings if $i<\eta$ in a single transmission. Thus, its transmission distance is given by

$$
x(i, w, \eta)= \begin{cases}\eta w, \quad i \geq \eta \\ i w, \quad \text { otherwise }\end{cases}
$$

In each data cycle, a sensor in $C_{i}$ transmits self-generated $\lambda_{s}$ data as well as relay $I_{s}(i, w, \eta)$ traffic. Thus, the energy consumption is

$$
\begin{aligned}
e_{s}(i, w, \eta)= & \left\{\alpha+\beta x^{\gamma}(i, w, \eta)\right\} \lambda_{s} \\
& +\left\{2 \alpha+\beta x^{\gamma}(i, w, \eta)\right\} I_{s}(i, w, \eta) \\
= & V(i, w, \eta) \times \lambda_{s},
\end{aligned}
$$

where $V(i, w, \eta)$ is the energy consumption by a sensor in ring $C_{i}$ per data cycle at unit data generation rate $\left(\lambda_{s}\right.$ is normalized to unity) and its value depends on the ring index $i$, ring thickness $w$, and hop size $\eta$ for given energy parameters $(\alpha, \beta$, and $\gamma)$ and network parameters $(R, \theta$, and $n$ ) and can be determined using (14), (15), and (16). The above equation leads to the following observations. For $i \geq \eta$, relay traffic $I_{s}(i, w, \eta)$ decreases with increasing ring index $i$ while transmission distance $\eta w$ remains same; hence the energy consumption by a sensor in ring $C_{\eta}$ is the maximum among sensors of rings $C_{\eta}$ to $C_{l}$. For sensors in rings $i \leq \eta$, incoming load $I_{s}(i, w, \eta)$ decreases and transmission distance $i w$ increases with the increasing ring index $i$. Due to these two opposite effects, the ring whose sensors have maximum energy consumption per data cycle among sensors in rings $C_{1}$ to $C_{\eta}$ depends on energy parameters $(\alpha, \beta)$, path loss factor $\gamma$, ring thickness $w$, and hop size $\eta$. However, for a given ring thickness $w$ and hop size $\eta$, the maximum per sensor energy consuming ring (i.e., the critical ring) is one among $C_{1}$ to $C_{\eta}$. In this regards, further analysis gives following theorem.

Theorem 1. Given a ring thickness $w$ and hop size $\eta(>1)$ :

1. for $\frac{\eta^{\gamma}-2 \eta+1}{\eta-1} \leq \frac{4 \alpha}{\beta w^{\gamma}}$, the critical ring is $C_{1}$; otherwise it is $C_{\eta}$. Both $C_{1}$ and $C_{\eta}$ become critical rings when the equality holds;

2. for $\frac{\eta^{\gamma}-2 \eta+1}{\eta-1}=\frac{4 \alpha}{\beta w^{\gamma}}$ and $\eta>2$, there exists a ring $C_{i}$, $(1<i<\eta)$ such that $e_{s}(1, w, \eta)>\cdots>e_{s}(i, w, \eta)$ and $e_{s}(i, w, \eta)<\cdots<e_{s}(l, w, \eta)$ and " $i$ " is obtained as the ceiling of the root of the equation $(\gamma-1) i^{\gamma}-$ $\frac{\gamma}{2} i^{\gamma-1}-\frac{2 \alpha}{\beta w^{\gamma}}=0$.

Proof. The proof is provided in Appendix A.

The above observation on energy distribution and critical ring, and Theorem 1 led us to develop the following transmission policies by formulating optimization problems to find the optimal ring thickness and hop size (or set of hop sizes) so that overall network lifetime is extended. The proposed transmission policies achieve increasingly uniform energy usage among sensors by exploiting varying degree of freedom in choosing hop sizes by sensors at various rings.

\subsection{Fixed Hop Size Transmission}

Considering cocentric rings around the sink, we propose a new approach by decomposing the transmission distance of 
traditional $\mathrm{MH}$ scheme into two parts: ring thickness and hop size pair, and a sensor forwards its data by the number of rings equal to the hop size at each transmission unlike only one ring in $\mathrm{MH}$ transmission. For a given pair $\mathbf{F}=[w, \eta]$, critical ring is either $C_{1}$ or $C_{\eta}$ but the value of critical energy varies with ring thickness $w$ and hop size $\eta$. Our goal is to find the optimal vector $\mathbf{F}_{\text {opt }}=[\hat{w}, \hat{\eta}]$ so that the critical energy is minimized and thus the overall network lifetime is maximized.

We assume that, $C_{1}$ is the critical ring and find the optimal vector $\mathbf{F}_{\text {opt }}=[\hat{w}, \hat{\eta}]$ to optimize critical energy consumed by a sensor in ring $\mathrm{C}_{1}$. Later we find that, for optimal vector $\mathbf{F}_{\text {opt }}=[\hat{w}, \hat{\eta}]$, energy consumption by a sensor in ring $C_{1}$ and $C_{\eta}$ are same. Thus, we get the following constraint for $C_{1}$ to be the critical ring (see Theorem 1):

$$
g(\mathbf{F})=\frac{\eta^{\gamma}-2 \eta+1}{\eta-1}-\frac{4 \alpha}{\beta w^{\gamma}} \leq 0 .
$$

Three more trivial constraints, as in (18), (19), and (20), that FHS transmission policy should satisfy are: 1) ring thickness $w$ should be greater than or equals to the minimum transmission range (see $r_{c o n}$ in (6)) required to maintain the network connectivity, 2) hop size be greater or equal to one, and 3) transmission distance of any sensor must not exceed the network radius $R$

$$
\begin{aligned}
& h(\mathbf{F})=r_{c o n}-w \leq 0, \\
& u(\mathbf{F})=1-\eta \leq 0, \\
& v(\mathbf{F})=\eta w-R \leq 0 .
\end{aligned}
$$

Substitution and simplification of (16) for a sensor in ring $C_{1}$ gives the critical energy that needs to be minimized and becomes our objective function

$$
f(\mathbf{F})=e_{s}(1, w, \eta)=\left(2 \alpha+\beta w^{\gamma}\right)(R+\eta w-w) \frac{R}{\eta w^{2}} \lambda_{s}-\alpha \lambda_{s} .
$$

The lifetime optimization using FHS transmission policy can thus be formulated as the following optimization problem:

$$
\begin{array}{cl}
\text { minimize } & f(\mathbf{F}) \\
\text { s.t. } & g(\mathbf{F}) \leq 0 ; h(\mathbf{F}) \leq 0 ; u(\mathbf{F}) \leq 0 ; \text { and } v(\mathbf{F}) \leq 0 .
\end{array}
$$

This is a standard nonlinear multiconstraints optimization problem that can be solved analytically using KKT (KarushKuhn-Tucker) conditions. If the constraints in (19) is active, i.e., $u(\mathbf{F})=0$, we have $\eta=1$, which is $\mathrm{MH}$ transmission. Similarly, if the constraints in (20) is active, i.e., $v(\mathbf{F})=0$, we have $\eta w=R$, which is effectively $\mathrm{SH}$ transmission. We already know their behavior from Section 3.3 and [14]. Therefore, for formulating and analyzing FHS policy, constraints (19) and (20) are assumed inactive, i.e., $u(\mathbf{F})<$ 0 and $v(\mathbf{F})<0$; and we minimize the cost function $f(\mathbf{F})$ with constraints $g(\mathbf{F}) \leq 0$ and $h(\mathbf{F}) \leq 0$. Using the KKT conditions, the solution to the optimization problem is

$$
\nabla f(\mathbf{F})+\mu_{1} \nabla g(\mathbf{F})+\mu_{2} \nabla h(\mathbf{F})=0,
$$

where $\mu_{1} \geq 0, \mu_{2} \geq 0$ and $\nabla f(\mathbf{F}), \nabla g(\mathbf{F})$, and $\nabla h(\mathbf{F})$ are the gradient vectors. Using the partial derivatives of the functions $f(\mathbf{F}), g(\mathbf{F})$, and $h(\mathbf{F})$ w.r.t $\eta$ in (23) we get

$$
\mu_{1}=\frac{R(R-w)\left(2 \alpha+\beta w^{\gamma}\right)(\eta-1)^{\gamma}}{n^{2} w^{2}\left\{(\gamma-1) \eta^{\gamma}-\gamma \eta^{\gamma-1}+1\right\}} \lambda_{s} .
$$

For (19) and (20) being inactive, i.e., $\eta>1$ and $\eta w<R$, and $2 \leq \gamma \leq 4$, the above equation yields $\mu_{1}>0$ and hence according to KKT theorem the constraint (17) is active. So,

$$
\begin{aligned}
& g(\mathbf{F})=\frac{\eta^{\gamma}-2 \eta+1}{\eta-1}-\frac{4 \alpha}{\beta w^{\gamma}}=0 \\
& \Rightarrow w=\left[\frac{4 \alpha(\eta-1)}{\beta\left(\eta^{\gamma}-2 \eta+1\right)}\right]^{\frac{1}{\gamma}}, \quad \eta>1 .
\end{aligned}
$$

From Theorem 1, we know that holding equality for constraint (17) leads to equal energy consumption per data cycle by a sensor in ring $C_{1}$ and $C_{\eta}$. Thus, the assumption of $C_{1}$ being the critical ring is right. Substitution of the value of $w$ as obtained in (24) in (21) gives a one variable relation consisting only hop size $\eta$ that may be solved analytically for optimal value that satisfy the constraints (18), (19), and (20). However, the solution becomes extremely complex, instead here we present a linear time algorithm, namely findOptimalParameters, to find the optimal values for $w$ and $\eta$ maintaining the constraints (18), (19), and (20) based on the relation obtained in (24).

\section{Algorithm. findOptimalParameters}

Step 1: Find a set of candidate vectors, denoted as $\mathbb{C}$, of ring thickness and hop size pair as follows:

$$
\begin{aligned}
& \mathbb{C}=\left\{\left[w_{\eta}, \eta\right] \mid\left[w_{\eta}, \eta\right] \text { holds }(24),\right. \\
& \left.\qquad \eta w_{\eta}<R \text { and } w_{\eta} \geq r_{\text {con }}\right\} . \\
& \text { If } \mathbb{C}=\varnothing \text { Then } \mathbb{C}=\left\{\left[r_{\text {con }}, 1\right]\right\} . \\
& \text { If } r_{\text {con }} \leq \hat{w}_{M H} \text { Then } \mathbb{C}=\mathbb{C} \cup\left\{\left[\hat{w}_{M H}, 1\right]\right\} . \\
& \mathbb{C}=\mathbb{C} \cup\{[R, 1]\} .
\end{aligned}
$$

The last two entries as union represent multihop and single-hop transmissions, respectively.

Step 2: Calculate the value of $V\left(1, w_{\eta}, \eta\right)$ (see (16)) for each vector $\left[w_{\eta}, \eta\right] \in \mathbb{C}$ which forms the the set

$$
\Lambda(\mathbb{C})=\left\{V\left(1, w_{\eta}, \eta\right) \mid\left[w_{\eta}, \eta\right] \in \mathbb{C}\right\} .
$$

Step 3: Find the optimal vector $\mathbf{F}_{\text {opt }}=[\hat{w}, \hat{\eta}]$ that gives the minimum value in $\Lambda(\mathbb{C})$.

\section{End}

Once the optimal vector, denoted by $\mathbf{F}_{\text {opt }}=[\hat{w}, \hat{\eta}]$, is decided, each sensor uses a fixed transmission range as determined by (15) (either $\hat{\eta} \hat{w}$ or $i \hat{w}$ ) throughout the entire lifetime, hence, called fixed hop size transmission. For optimal ring thickness $\hat{w}$ and hop size $\hat{\eta}$, the critical energy and network lifetime achievable in FHS transmission policy can be expressed as (25) and (26), respectively,

$$
\begin{aligned}
e_{c}(F H S) & =f\left(\mathbf{F}_{\text {opt }}\right) \\
& =\frac{\left(2 \alpha+\beta \hat{w}^{\gamma}\right)(R+\hat{\eta} \hat{w}-\hat{w}) R \lambda_{s}}{\hat{\eta} \hat{w}^{2}}-\alpha \lambda_{s} \\
L_{f h s} & =E_{\text {init }} / e_{c}(F H S) .
\end{aligned}
$$

Distributed implementation and complexity. At the network set up phase, sink broadcasts network parameters (sector radius $R$, angle at $\operatorname{sink} \theta$, number of sensors $n$, desired connectivity probability $p_{c o n}$, and sink location) to all sensors. Since energy parameters $(\alpha, \beta$, and $\gamma)$ are known, a sensor calculates the value of $r_{\text {con }}$ using (6) and determine the set $\mathbb{C}$ locally (using (24)) by increasing hop size gradually. For each 
candidate pair $\left[w_{\eta}, \eta\right] \in \mathbb{C}$, a sensor calculate the value of $V\left(1, w_{\eta}, \eta\right)$ in Step 2 and hence determine their minimum value in Step 3 to identify optimal ring thickness and hop size pair. Using (24), we can show that, as the value of $\eta$ increases, $w_{\eta}$ decreases and their product $\eta w_{\eta}$ increases. Thus, the maximum hop size $\eta_{\max }$ is finite and is bounded by the constraints $\eta_{\max } w_{\eta_{\max }}<R$ and $w_{\eta_{\max }} \geq r_{\text {con }}$, and the overall complexity of the algorithm is $\Theta\left(\eta_{\max }\right)$.

Sensor density requirement. In determining the optimal ring thickness and hop size pair, algorithm findOptimalParameters considers the minimum transmission distance $\left(r_{c o n}\right)$ required to maintain node connectivity which, in turn, depends on the number of sensors $n$. As the number of sensors deployed reduces, according to (6), the value of $r_{c o n}$ increases, and hence, lower candidate vectors satisfy $w_{\eta} \geq$ $r_{c o n}$ in the Step 1 of the algorithm and eventually finds optimal pair among them in Step 3. Thus, FHS transmission scheme is adaptive with node density.

Performance analysis shows that, network lifetime improves significantly in FHS transmission over $\mathrm{SH}, \mathrm{MH}$, and their hybrid transmission policies presented in [14].

\subsection{Synchronous Variable Hop Size Transmission}

Although optimally obtained vector $\mathbf{F}_{\text {opt }}=[\hat{w}, \hat{\eta}]$ in FHS transmission minimizes the critical energy and improves network lifetime, the following observation in Theorem 2 suggests that further improvement is possible by varying hop size over the network lifetime.

Theorem 2. For a given ring thickness $w$, the energy consumption per sensor per data cycle in the higher indexed rings $(i>\eta)$ become higher and in lower indexed rings $(i \leq \eta)$ become lower with the increase in hop size. Thus,

$$
\begin{aligned}
& \text { 1. } e_{s}(i, w, \eta+1)<e_{s}(i, w, \eta) \text { for } i \leq \eta \text { and } \\
& \text { 2. } e_{s}(i, w, \eta+1)>e_{s}(i, w, \eta) \text { for } i>\eta .
\end{aligned}
$$

Proof. The proof is provided in Appendix B.

Therefore, the hop size plays a vital role in determining the ring whose two sides exhibit two distinct behaviors: reduces energy consumption for inward side and increases energy consumption for outward side with increasing hop size. This leads to a logical conclusion that, varying hop size over lifetime instead of keeping it fixed would result in better distribution of energy usage; hence better network lifetime is expected. In SVHS transmission policy, sensors in all rings concomitantly use the same hop size for the same number of data cycles but the hop size changes over lifetime.

For analysis purpose, SVHS uses the same network and traffic model as used in FHS transmission. The whole sector is divided into $\hat{l}=\lceil R / \hat{w}\rceil$ cocentric rings with optimal ring thickness $\hat{w}$ as obtained in FHS transmission. Let $\mathbf{\Phi}=$ $[\Phi(1), \Phi(2), \ldots, \Phi(\hat{l})]^{\mathrm{T}}$ be a vector with $\Phi(j)$ as the duty cycle, number of data cycles a sensor use a particular hop size $j$, $1 \leq j \leq \hat{l}$ over the entire lifetime and $B(i, j)$ be the energy consumption by a sensor in ring $C_{i}$ per data cycle to transmit its self-generated data, $\lambda_{s}$ and relay incoming traffic, $I_{s}(i, \hat{w}, j)$ while hop size is $j$. Thus, we have the following energy matrix:

$$
\mathbf{B}=[B(i, j)]_{\hat{l} \times \hat{l}}, \quad \text { where } B(i, j)=e_{s}(i, \hat{w}, j) .
$$

The total energy usage by a sensor in ring $C_{i}$ for the vector $\Phi$ can be calculated as (28) and must be less than or equal to the initial sensor energy, $E_{\text {init }}$. Thus, we have a set of $\hat{l}$ constraints as in (29) that must be satisfied while finding optimal $\boldsymbol{\Phi}$ in SVHS transmission

$$
\begin{aligned}
e(i, \boldsymbol{\Phi}) & =\sum_{j=1}^{\hat{l}} \Phi(j) B(i, j), \\
Q(\boldsymbol{\Phi}) & =\mathbf{B} \boldsymbol{\Phi}-\mathbf{I} E_{\text {init }} \leq \mathbf{0},
\end{aligned}
$$

where $\mathbf{I}$ is an identity vector. Another trivial constraint SVHS should satisfy is that, duty cycle for any hop size, i.e., $\Phi(j)$ s must be greater than or equals to zero. So,

$$
\boldsymbol{\Phi} \geq \mathbf{0} .
$$

The network lifetime in SVHS transmission, denoted as $L_{s v h s}$, for a particular vector of hop sizes $\boldsymbol{\Phi}$ is

$$
L_{s v h s}=\|\boldsymbol{\Phi}\|_{1}=\sum_{j=1}^{\hat{l}} \Phi(j) .
$$

Our goal is to maximize $L_{s v h s}$ by choosing an optimal vector, $\hat{\boldsymbol{\Phi}}$. Thus, lifetime maximization using SVHS transmission policy can be formulated as

$$
\begin{array}{cl}
\operatorname{maximize} & L_{\text {svhs }} \\
\text { s.t. } & Q(\boldsymbol{\Phi}) \leq \mathbf{0}, \\
& \mathbf{\Phi} \geq \mathbf{0} .
\end{array}
$$

The solution of the above optimization problem using KKT theorem gives (see Appendix C):

$$
\Phi(i)=z_{i}-\sum_{j=i+1}^{\hat{l}} \Phi(j) U(i, j), \quad 1 \leq i \leq \hat{l},
$$

where $U(i, j)$ and $z_{i}$ are given by $(c 4)$ and $(c 7)$ in Appendix $C$, respectively. Moreover, duty cycle for any hop size is the number of complete data cycles and thus must be an integer number. Hence,

$$
\hat{\Phi}(i)=\lfloor\Phi(i)\rfloor, \quad 1 \leq i \leq \hat{l} .
$$

The performance of SVHS transmission policy as illustrated in Section 6 shows further improvement in network lifetime over FHS transmission and energy usage among sensor nodes are almost balanced.

\subsection{Asynchronous Variable Hop Size Transmission}

While SVHS transmission achieves better energy distribution and longer lifetime than other policies, all sensors use the same hop size at a given instance and the same set of hop sizes over the entire lifetime. Since each ring's distance from the sink is different, it is more logical and a better energy and load distribution is expected by using a distinct set of hop sizes for each ring. Based on this idea, we propose AVHS transmission policy where each ring employs a distinct set of optimal hop sizes and associated duty cycles over lifetime. In the rest of this section, we formulate traffic and energy consumption model differently for AVHS transmission.

Let $\Im(i, j)$ be the number of data cycles (sends $\lambda_{s}$ data at each cycle) a sensor in ring $C_{i}$ use hop size $j(1 \leq j \leq i)$ over 
the entire lifetime. A sensor in the outermost ring $\left(C_{\hat{l}}\right)$ transmits its self-generated data only. Maintaining continuous flow of incoming and outgoing data at each ring, lifetime is the time for which the data generated in ring $C_{\hat{l}}$ is serviced through all the way to the sink. Therefore, the number of data cycles in AVHS transmission policy, i.e., the network lifetime $L_{a v h s}$ is equal to the total number of completed data cycles by a sensor in ring $C_{\hat{l}}$ and is

$$
L_{\text {avhs }}=\sum_{j=1}^{\hat{l}} \Im(\hat{l}, j) .
$$

The hop size $j$ is $\leq i$ for a sensor in ring $C_{i}$; since any hop size greater than $i$ is merely a wastage of energy. Then the total data transmitted by all sensors in ring $C_{i}$ over the entire lifetime are

$$
\Gamma(i, \hat{w})=\sum_{j=1}^{i} N(i, \hat{w}) \Im(i, j) \lambda_{s}, \quad 1 \leq i \leq \hat{l},
$$

where $\hat{w}$ is obtained as in Section 4.3 and $\hat{l}=\lceil R / \hat{w}\rceil$. Data transmitted by sensors in ring $C_{i+j}$ using hop size $j$ are eventually received in ring $C_{i}$. The traffic coming from all sensors in ring $C_{i+j}$ to the sensors in ring $C_{i}$ over the entire lifetime is $N(i+j, \hat{w}) \Im(i+j, j) \lambda_{s}$. Then, the total incoming traffic on all sensors in ring $C_{i}$ from all outer rings over the entire network lifetime is given by

$$
\chi(i, \hat{w})= \begin{cases}\sum_{k=i+1}^{\hat{l}} N(k, \hat{w}) \Im(k, k-i) \lambda_{s}, & 1 \leq i<\hat{l}, \\ 0, & i=\hat{l} .\end{cases}
$$

Data generated by all $N(i, \hat{w})$ sensors in ring $C_{i}$ over network lifetime of $L_{\text {avhs }}$ data cycles are

$$
\wp(i, \hat{w})=L_{\text {avhs }} N(i, \hat{w}) \lambda_{s} .
$$

Thus, the total traffic that needs to be serviced by all sensors in ring $C_{i}$ throughout its entire life is

$$
\Re(i, \hat{w})=\chi(i, \hat{w})+\wp(i, \hat{w}) .
$$

In order to maintain flow equilibrium, the sum of incoming traffic and self-generated data, $\Re(i, w)$ in (39), must be equal to the total transmitted data, $\Gamma(i, \hat{w})$ in (36), for each ring over the network. Thus,

$$
\Gamma(i, \hat{w})=\Re(i, \hat{w}), \quad 1 \leq i \leq \hat{l} .
$$

From (36) to (40), we get the following $\hat{l}$ constraints, namely, flow constraints:

$$
\begin{aligned}
& G_{k}(\Im)=\sum_{j=1}^{k} \Im(k, j)-\sum_{j=1}^{\hat{l}} \Im(\hat{l}, j) \\
& \quad-\frac{1}{N(k, \hat{w})}\left[\sum_{j=k+1}^{\hat{l}}\{N(j, \hat{w}) . \Im(j, j-k)\}\right]=0, \quad 1 \leq k \leq \hat{l} .
\end{aligned}
$$

Altogether $N(i, \hat{w})$ sensors in ring $C_{i}$ receive $\chi(i, \hat{w})$ incoming traffic from the outer rings during the entire lifetime, on average the energy consumed by an individual sensor of ring $C_{i}$ to receive incoming data is

$$
e_{a}^{r}(i, \hat{w}, \Im)=\frac{\chi(i, \hat{w})}{N(i, \hat{w})} \alpha .
$$

The energy consumed by a sensor in ring $C_{i}$ for transmitting data using hop size $j$ for $\Im(i, j)$ number of data cycles and the total energy for data transmission over the entire network lifetime are given by

$$
\begin{aligned}
e_{a}^{t}(i, \hat{w}, j) & =\Im(i, j)\left\{\alpha+\beta x^{\gamma}(i, \hat{w}, j)\right\} \lambda_{s}, \text { and } \\
e_{a}^{t}(i, \hat{w}, \Im) & =\sum_{j=1}^{i} e_{a}^{t}(i, \hat{w}, j) .
\end{aligned}
$$

The total energy consumed by a sensor of ring $C_{i}$ for both reception and transmission of data throughout its lifetime is $e_{a}^{r}(i, \hat{w}, \Im)+e_{a}^{t}(i, \hat{w}, \Im)$ which must be less than or equal to the initial energy storage, $E_{\text {init }}$; hence, simplification using (37), (42), and (43) gives the following $\hat{l}$ constraints, namely, energy constraints:

$$
\begin{aligned}
H_{k}(\Im)= & \sum_{j=1}^{k}\left[\Im(k, j) .\left\{2 \alpha+\beta(x(k, \hat{w}, j))^{\gamma}\right\} \lambda_{s}\right] \\
& -\sum_{j=1}^{\hat{l}} \Im(\hat{l}, j) \alpha \lambda_{s}-E_{\text {init }} \leq 0, \quad 1 \leq k \leq \hat{l} .
\end{aligned}
$$

Our objective is to determine the distinct set of hop sizes ( $\Im)$ used for sensors in each ring that maximizes the overall network lifetime $L_{a v h s}$ and satisfies the flow and energy constraints in (41) and (44). Thus, the following optimization problem represents AVHS transmission:

$$
\begin{aligned}
\operatorname{maximize} & L_{\text {avhs }} \\
\text { s.t. } & H_{k}(\Im) \leq 0, \quad 1 \leq k \leq \hat{l}, \\
& G_{k}(\Im)=0, \quad 1 \leq k \leq \hat{l} .
\end{aligned}
$$

We can convert the above optimization problem into the following standard LP form that can be solved in polynomial time:

$$
\begin{aligned}
\operatorname{maximize} & \mathbf{c}^{\mathrm{T}} \mathbf{X} \\
\text { s.t } & \mathbf{A X}=\mathbf{b}, \\
& \mathbf{X} \geq \mathbf{0},
\end{aligned}
$$

where $\mathbf{A}, \mathbf{X}, \mathbf{c}$, and $\mathbf{b}$ are defined below.

$\mathbf{X}=\left[x_{1}, x_{2}, \ldots, x_{p}, x_{p+1}, \ldots, x_{q}\right]^{T}$ is a vector with $x_{1}$ to $x_{p}$ are the decision variables and $x_{p+1}$ to $x_{q}$ are the slake variables and defined as

$$
x_{m}= \begin{cases}\Im(i, j), & 1 \leq m \leq p, \\ 0, & p+1 \leq j \leq q,\end{cases}
$$

where $p=\hat{l}(\hat{l}+1) / 2, q=p+\hat{l}, 1 \leq i \leq \hat{l}, 1 \leq j \leq i$, and $m=i(i-1) / 2+j$.

$\mathbf{c}=\left[c_{1}, c_{2}, \ldots, c_{q}\right]^{\mathbf{T}}$ is the coefficient vector of the objective function defined as

$$
c_{i}= \begin{cases}1, & p-\hat{l}+1 \leq i \leq p \\ 0, & \text { otherwise }\end{cases}
$$

$\mathbf{b}=\left[b_{1}, b_{2}, \ldots, b_{2 i}\right]^{\mathbf{T}}$ is the right hand side vector of the constraints where

$$
b_{i}= \begin{cases}E_{\text {init }}, & 1 \leq i \leq \hat{l}, \\ 0, & \text { otherwise }\end{cases}
$$


$\mathbf{A}=\left[a_{i j}\right]_{2 \hat{l} \times q}$ is the coefficient matrix for all $2 \hat{l}$ constraints (energy constraint $H_{k}(\Im) \mathrm{s}$ are numbered as 1 to $\hat{l}$ and flow constraint $G_{k}(\Im) \mathrm{s}$ are numbered as $\hat{l}+1$ to $\left.2 \hat{l}\right)$ with $a_{i j}$ being the coefficient of $x_{j}$ in the $i$ th constraint and can be defined as

For $1 \leq i<\hat{l}$ :

$a_{i j}=\left\{\begin{array}{lc}{\left[2 \alpha+\beta\left\{x(i, \hat{w}, m\}^{\gamma}\right] \lambda_{s},\right.} & j=i(i-1) / 2+m, \\ & 1 \leq m \leq i, \\ -\alpha \lambda_{s}, & \hat{l}(\hat{l}-1) / 2+1 \leq j \\ 1, & \leq \hat{l}(\hat{l}+1) / 2, \\ 0, & j=p+i, \\ & \text { otherwise, }\end{array}\right.$

$a_{(\hat{l}+i) j}= \begin{cases}1, & i(i-1) / 2+1 \leq j \\ & \leq i(i+1) / 2, \\ -1-N(\hat{l}, \hat{w}) / N(i, \hat{w}), & j=\hat{l}(\hat{l}+1) / 2-i, \\ -1, & \hat{l}(\hat{l}-1) / 2+1 \leq j \\ & \leq \hat{l}(\hat{l}+1) / 2 \\ & \text { and } j \neq \hat{l}(\hat{l}+1) / 2-i, \\ -N(i+m, \hat{w}) / N(i, \hat{w}), & i<\hat{l}-1,1 \leq m \\ & \leq \hat{l}-i-1, \\ & \text { and } j=(i+m) \\ & (i+m-1) / 2+m, \\ 0, & \text { otherwise. }\end{cases}$

For $i=\hat{l}$ :

$a_{\hat{l} j}=\left\{\begin{array}{lc}{\left[2 \alpha+\beta x(\hat{l}, \hat{w}, m)^{\gamma}\right] \lambda_{s}-\alpha \lambda_{s},} & j=\hat{l}(\hat{l}-1) / 2+m, \\ 1, & \text { and } 1 \leq m \leq \hat{l}, \\ j=p+\hat{l}, & \text { otherwise. }\end{array}\right.$

For $i=2 \hat{l}: a_{(2 \hat{l}) j}=0, \quad 1 \leq j<q$.

In AVHS transmisison, network lifetime improves significantly compared to the transmission policies in [14], FHS, and SVHS, and energy usage distribution is completely balanced over the network (see Section 6).

Message Overhead. At the network set up phase, sink determines the optimal parameters (ring thickness, hop sizes, and their associated duty cycles) based on the collected information regarding sensor organization/topology and then disseminate them among sensors. In SVHS transmission, sink broadcasts one real and $2 l$ number of integer values and in AVHS transmission sink broadcasts one real and $l(l+1)$ integer values. The amount of data transmission for this purpose is very low compared to the volume of traffic transmitted in very few data cycles. Moreover, in many applications, sensors could be preprogrammed before deployment with these optimal values, calculated in advance from predefined network parameters.

\section{Distributed HeURistics for SVHS AND AVHS TRANSMISSIONS}

In this section, we present heuristics for the distributed implementation of SVHS and AVHS transmission policies proposed in Section 4. First, we analyze the distribution of energy usage among sensors for varying ring thickness and hop size. Such insights identify some inherent patterns which led us to develop relation among hop sizes and their duty cycles that maximizes the network lifetime. In the following, we define a list of parameters related to the heuristics:

- $\quad E_{\max }\left(w_{z}, j\right)$ : the critical (maximum) energy consumption by a sensor per data cycle for ring thickness and hop size pair $\left[w_{z}, j\right]$ over all rings. Here, $w_{z}$ is the ring thickness given by (24) for hop size $z>1$, or $\hat{w}_{M H}$ as in (11) for $z=1$ and $\gamma>2$, or $R$ for $z=1$ and $\gamma=2$. According to Theorem 1, for ring thickness and hop size pair $\left[w_{z}, j\right]$, critical ring is either $C_{1}$ or $C_{j}$, hence

$$
\begin{aligned}
E_{\max }\left(w_{z}, j\right) & =\max \left\{e_{s}\left(1, w_{z}, j\right), e_{s}\left(j, w_{z}, j\right)\right\} \\
& =\max \left\{V\left(1, w_{z}, j\right), V\left(j, w_{z}, j\right)\right\} \times \lambda_{s} .
\end{aligned}
$$

- $\quad E_{\min }\left(w_{z}, j\right)$ : the minimum energy consumption by a sensor per data cycle for the pair $\left[w_{z}, j\right]$ over all rings. For $\left[w_{z}, j\right]$, the minimum energy consuming ring between $C_{1}$ to $C_{j}$ is determined as the root of the equation given in Theorem 1(b) and, hereafter, the corresponding ring index be denoted by $i_{\min }\left(w_{z}\right)$. For rings $C_{i}$ with $i \geq j$, the energy consumption decreases as ring index increases, since relay traffic decreases while transmission distance remains same. For hop size $j$, sensors in rings $C_{l_{z}-j+1}$ to $C_{l_{z}}$ have no relay traffic and among them $C_{l_{2}-j+1}$ could have the minimum transmission distance depending on its hop distance from sink. So,

$$
\begin{aligned}
E_{\min }\left(w_{z}, j\right)= & \min \left\{e_{s}\left(i_{\min }\left(w_{z}\right), w_{z}, j\right),\right. \\
& \left.e_{s}\left(l_{z}-j+1, w_{z}, j\right)\right\} \\
= & \min \left\{V\left(i_{\min }\left(w_{z}\right), w_{z}, j\right),\right. \\
& \left.V\left(l_{z}-j+1, w_{z}, j\right)\right\} \times \lambda_{s},
\end{aligned}
$$

where $l_{z}$ is the total number of rings for ring thickness $w_{z}$.

- $D\left(w_{z}, j\right)$ : the maximum deviation in energy consumption for the ring thickness and hop size pair $\left[w_{z}, j\right]$. Such a deviation is a metric for the nonuniformity in energy usage among sensors and is given by

$$
D\left(w_{z}, j\right)=E_{\max }\left(w_{z}, j\right)-E_{\min }\left(w_{z}, j\right) .
$$

Before the development of heuristics, we illustrate the maximum energy usage deviation among sensors for different ring thickness and hop size pairs with example. For ring thickness $w_{z}, D\left(w_{z}, j\right) / D\left(w_{z}, z\right)$ represents the relative deviation in energy consumption when hop size $j$ is used compared to hop size $z$. Fig. 2 shows relative energy usage deviation with four different ring thicknesses $\left(w_{3}, w_{4}\right.$, $w_{5}$, and $w_{6}$ ) by varying hop sizes. All four cases show that, deviation is the minimum when hop size $z$ is used with ring thickness $w_{z}$ as determined in (24). As the hop size $j$ changes either direction, the deviation in the maximum and minimum energy usage increases making energy usage among sensors more nonuniform. Based on this observation, in the following, we propose heuristics for SVHS and AVHS transmissions. 


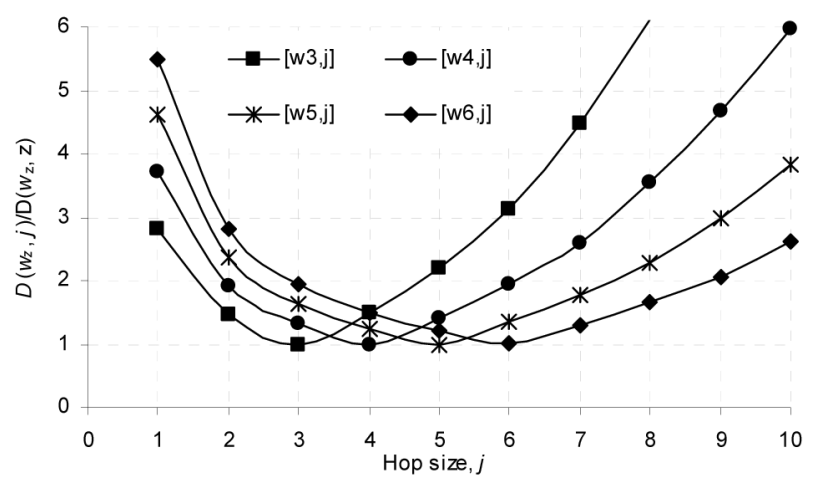

Fig. 2. The relative deviation in maximum and minimum energy usage among sensors $\left(R=1,000 \mathrm{~m}, \lambda_{\mathrm{s}}=525\right.$ bytes, and $\left.\gamma=4\right)$.

Heuristic for SVHS Transmission. As described in Section 4.4, in SVHS transmission, optimal ring thickness $(\hat{w})$ is obtained as a part of the optimal vector $\mathbf{F}_{\text {opt }}=[\hat{w}, \hat{\eta}]$. The aim in SVHS transmission is to attain uniform energy usage among sensors by varying hop sizes for which the maximum deviation $D(\hat{w}, j)$ is a measure of nonuniformity. The higher is the value of $D(\hat{w}, j)$ compare to $D(\hat{w}, \hat{\eta})$, the more nonuniform is energy usage among sensors and thus the lower is the number of data cycles to use hop size $j$, $1 \leq j \leq \hat{l}$. Moreover, according to Theorem 1, for ring thickness $\hat{w}$, as the hop size $j$ reduces from $\hat{\eta}$, ring $C_{1}$ become the sole critical ring and according to Theorem 2, energy usage by a sensor in ring $C_{1}$ increases whereas the energy usage in the outer rings $\left(C_{i}, i>\hat{\eta}\right)$ decreases. Hence, greater $D(\hat{w}, j)$ and the relatively small number of sensors in $C_{1}$ overdrain their energy quickly which is highly undesirable. On the other hand, as the hop size $j$ increases from $\hat{\eta}$, ring $C_{\hat{\eta}}$ become the sole critical ring. Although the energy consumption at the inner rings reduces, the critical energy increases very fast. Based on the above observations, we define following proportionality for the duty cycle of hop size $j$ :

$$
\Phi(j) \propto \begin{cases}0, & j<\hat{\eta} \\ \frac{1}{D(\hat{w}, j)} \times \frac{1}{|j-\hat{\eta}|+1}, & j \geq \hat{\eta} .\end{cases}
$$

By replacing $j=\hat{\eta}$, we find the proportionality constant as $\Phi(\hat{\eta}) D(\hat{w}, \hat{\eta})$. Thus,

$$
\Phi(j): \Phi(\hat{\eta})=\frac{D(\hat{w}, \hat{\eta})}{D(\hat{w}, j)} \times \frac{1}{|j-\hat{\eta}|+1}, \quad j \geq \hat{\eta} .
$$

From (47) to (50), it is easily observed that duty cycle ratio $\Phi(j): \Phi(\hat{\eta})$ is independent of $\lambda_{s}$ and can be calculated in a distributed manner by sensors knowing energy parameters and minimal network parameters, namely, $R$, $\theta, n$, and $p_{c o n}$. The duty cycle ratio for each hop size can be determined in $\Theta(1)$ time, and thus the overall complexity to find ratios for $\hat{l}$ hops is $\Theta(\hat{l})$. Since the value of the number of rings $\hat{l}$ is usually small, $\Theta(\hat{l})$ is reasonably a low complexity.

Heuristic for AVHS Transmission. Unlike SVHS transmission, different set of hop sizes are used by sensors located in different rings in AVHS transmission. A sensor in ring $C_{i}$ uses hop sizes 1 to $i$ and $\Im(i, j)$ is the duty cycle for hop size $j, 1 \leq j \leq i$. Here, we would consider two
TABLE 1

System Parameters

(i) Network parameters

Network radius, $R$

$1000 m$

Number of sensors, $n$

$10^{5}$

Node con. prob., $p_{c o n}$

0.99

Average data rate, $\lambda_{s}$

525 bytes/data cycle

(ii) Energy parameters

$$
\begin{aligned}
& 2 \leq \gamma \leq 4 \\
& a=50 \mathrm{~nJ} / \mathrm{bit} \\
& \beta=10 \mathrm{pJ} / \mathrm{bit} / \mathrm{m}^{2} \text { for } \gamma=2 \\
& =0.0013 \mathrm{pJ} / \mathrm{bit} / \mathrm{m}^{4} \text { for } \gamma=4
\end{aligned}
$$

phenomena in determining $\Im(i, j): 1)$ how good hop $j$ is in terms of overall energy usage distribution $(D(\hat{w}, j)$ value should be lower), and 2) how good it is for a sensor in ring $C_{i}$ (energy usage $e_{s}(i, \hat{w}, j)$ should be lower). Despite a hop is very energy efficient for sensors in ring $C_{i}$, it should not be used too frequently if its overall energy behavior is very nonuniform and vice versa. To meet the above criteria, we define $\Im(i, j)$ as follows:

$$
\Im(i, j) \propto \begin{cases}0, & j<\min (i, \hat{\eta}), \\ \frac{1}{D(\hat{w}, j)} \times \frac{1}{e_{s}(i, \hat{w}, j)+e_{s}(i, \hat{w}, \hat{\eta})}, & j \geq \min (i, \hat{\eta}) .\end{cases}
$$

Finding the proportionality constant with $j=\hat{\eta}$ gives

$$
\begin{aligned}
\Im(i, j): \Im(i, \hat{\eta})= & \frac{2 e_{s}(i, \hat{w}, \hat{\eta})}{e_{s}(i, \hat{w}, j)+e_{s}(i, \hat{w}, \hat{\eta})} \times \frac{D(\hat{w}, \hat{\eta})}{D(\hat{w}, j)} \\
= & \frac{2 V(i, \hat{w}, \hat{\eta})}{V(i, \hat{w}, j)+V(i, \hat{w}, \hat{\eta})} \times \frac{D(\hat{w}, \hat{\eta})}{D(\hat{w}, j)}, \\
& j \geq \min (i, \hat{\eta}) .
\end{aligned}
$$

Similar to (50), the above ratio is also independent of $\lambda_{s}$ and a sensor can determine (51) in a distributed manner in $\Theta(1)$ time. A sensor in ring $C_{i}$ computes a total of $i$ hop sizes, hence the complexity for a ring $C_{i}$ sensor to find all duty cycle ratios is $\Theta(i), 1 \leq i \leq \hat{l}$.

\section{Performance Evaluation}

In this section, we present some illustrative numerical results to assess the performance of the proposed transmission policies. The system parameters we used for the computational results in this section are the same as in [14] and are listed in Table 1 . We divide our numerical results into two parts: 1) distribution of energy usage among sensors with ring thickness and hop size in the proposed traffic model, and 2) comparison of proposed transmission policies with the existing $\mathrm{SH}, \mathrm{MH}$, and their hybrid transmission schemes in terms of optimal lifetime and energy usage among sensors. Here, we present the result for higher path loss factor $(\gamma=4)$, since WSNs are usually deployed in hostile terrain. It may be noted that, improvements are even better for low path loss factor $(\gamma=2)$, but not shown here for space limitation. 


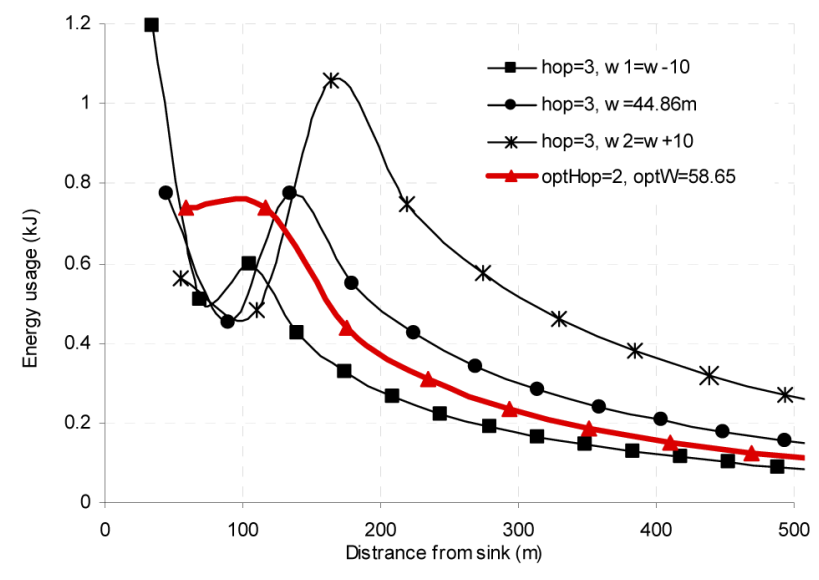

Fig. 3. Effect of changing ring thickness on the energy usage by sensors located at various distances from the sink for $10^{4}$ data cycles $(\gamma=4)$.

\subsection{Distribution of Energy Usage among Sensors with Ring Thickness and Hop Size}

Here, we illustrate how critical ring and the value of critical energy vary with the choice of ring thickness and hop size. Fig. 3 shows the effect of changing ring thickness on the energy usage by sensors located at various distances from the sink for hop size $\eta=3$ with $\gamma=4$. The ring thickness obtained for $\eta=3$ according to (24) is $w=44.86 \mathrm{~m}$ and critical ring is either $C_{1}$ or $C_{3}$, and the energy consumed by a sensor in the critical ring for $10^{4}$ data cycles is $776 \mathrm{~J}$ (two peaks of the circular legend line). Reducing the ring thickness to $w 1=w-10$ makes $C_{1}$ the sole critical ring and the critical energy increases to $1196.5 \mathrm{~J}$ while increasing ring thickness to $w 2=w+10$ makes $C_{3}$ the sole critical ring with $1060 \mathrm{~J}$ as critical energy. This observation establishes the fact that, for any given hop size, the ring thickness as determined by (24) gives the minimum critical energy. Moreover, for the system parameters as listed in Table 1, Algorithm 1 yields optimal vector (ring thickness and hop size pair) $\mathrm{F}_{\text {opt }}=[58.65 \mathrm{~m}, 2]$ for path loss factor $\gamma=4$. The solid bold lines in Fig. 3 shows that critical energy $739.0 \mathrm{~J}$ is the minimum when optimal hop size is in use. From Fig. 3 we also observe that, for fixed hop size, energy usage by sensors in the critical ring becomes the bottleneck for the overall network lifetime.

Fig. 4 shows the effect of varying hop size on the location of critical ring and the critical energy for a given ring

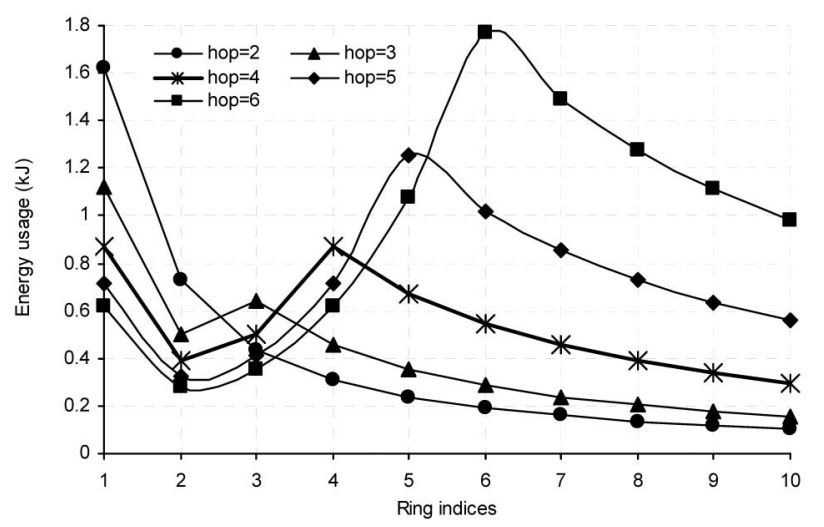

Fig. 4. Effect of changing hop size on energy usage by sensors at various rings for $10^{4}$ data cycles for $w=36.9 \mathrm{~m}$ and $\gamma=4$.

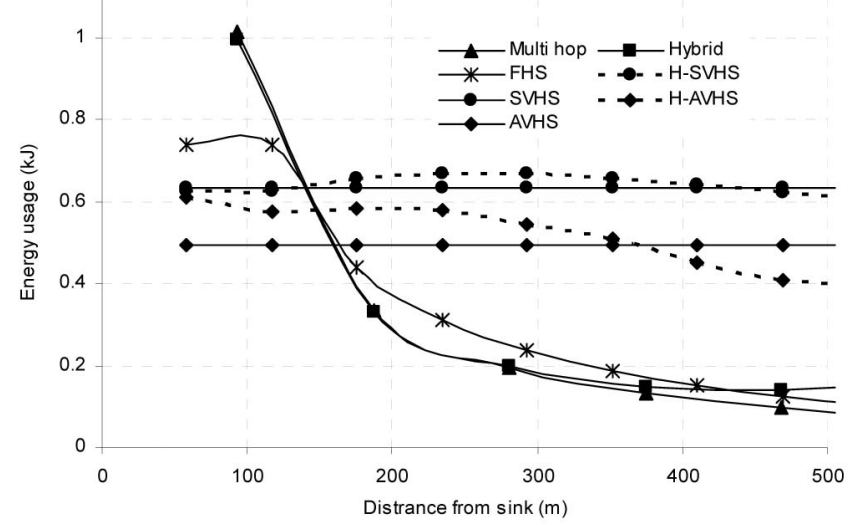

Fig. 5. Distribution of energy usage among sensors located at various distances from the sink for $10^{4}$ data cycles.

thickness. We vary hop size, $\eta$ from 2 to 6 for ring thickness $w=36.9 \mathrm{~m}$ (obtained from (24) for hop size $\eta=4$ ). Critical ring is both $C_{1}$ and $C_{4}$ for $\eta=4, C_{1}$ for $\eta<4$, and $C_{\eta}$ for $\eta>4$; hence comply with Theorem 1 . Moreover, critical energy is minimum (868.9 J) for hop size 4 among the critical energies because we used ring thickness corresponding to hop size 4 . Further details reveal the trend in change in energy consumption by sensors in different rings with changing hop size. For example, when we change hop size from 4 to 5 , for sensors in rings 1 to 4 , energy consumption per data cycle decreases and for sensors in rings above 4 ( 5 to $l$ ) energy consumption increases. Similar trend is maintained for other hop size changes as well. This observation verifies Theorem 2 .

\subsection{Comparison of Optimal Lifetime and Energy Usage}

Fig. 5 shows the distribution of optimal energy usage among sensors having varying distances from the sink in different transmission policies for path loss factor $\gamma=4$. For system parameters as listed in Table 1, critical energy in FHS transmission (739.4 J) is significantly lower than SH $(54600 \mathrm{KJ})$ (not shown here), MH (1014.3 J) and their hybrid $(996.0 \mathrm{~J})$ transmissions. For SVHS transmission, critical energy is even lower and energy distribution among sensors is almost uniform with maximum and minimum energy consumptions being 633.2 and $629.6 \mathrm{~J}$. Critical energy $(493.2 \mathrm{~J})$ is the lowest for AVHS transmission policy and energy usage is completely balanced among sensors. Fig. 5 also shows the distribution of energy usage for H-SVHS and H-AVHS transmissions (shown in dotted lines). Numerical results show that, performance of H-SVHS closely follows centralized SVHS while that of H-AVHS attains reasonable match with AVHS transmission.

Fig. 6 compares lifetime achievable in different transmission policies for varying initial sensor energy. Results show that, hybrid of single and multihop transmissions as presented in [14] improves network lifetime only insignificantly. When the proposed transmission policies are employed, improvement in lifetime over $\mathrm{MH}$ is significant; above 130 percent for FHS, above 150 percent for SVHS, and above 200 percent for AVHS transmission policies for $\gamma=4$. Improvements in H-SVHS and H-AVHS transmission policies are also significant, and above 150 and 165 percent, 


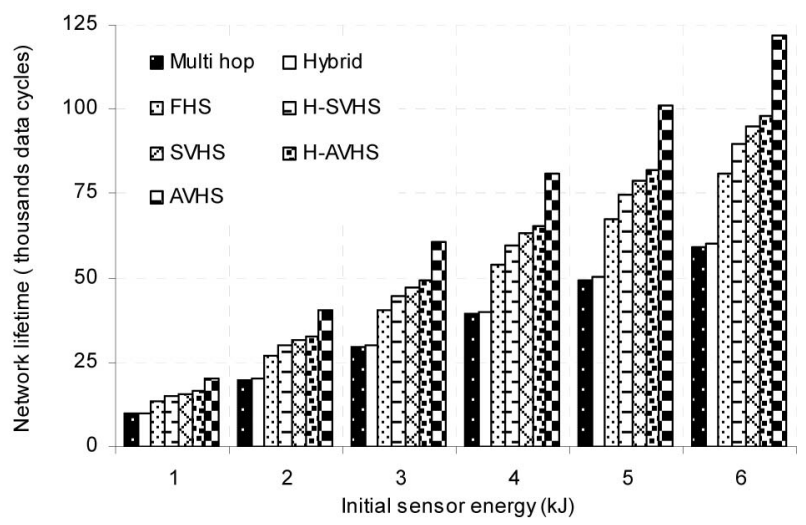

Fig. 6. Network lifetime with various initial sensor energy $(\gamma=4)$.

respectively, over multihop transmission. We also found that, for $\gamma=2$, the improvements in FHS, SVHS, and AVHS are 200, 250, and 300 percent, respectively, over MH.

Finally, we investigated the effect of network size on lifetime by varying the network radius and the results are presented in Fig. 7. Here, we plotted the ratio of the lifetime achievable in Hybrid, FHS, H-SVHS, SVHS, H-AVHS, and AVHS transmission policies w.r.t. MH transmission. With the same initial energy and sensor density, network lifetime decreases exponentially with the increase in network radius as more data need to be relayed by a sensor at each data cycle. But network lifetime achievable by using FHS, HSVHS, SVHS, H-AVHS, and AVHS transmission policies maintain their superiority in comparison to others irrespective of the network size.

\section{Conclusions}

In this paper, we propose three transmission policies that achieve increasingly extended network lifetime through improved distribution of energy usage among sensors. The FHS scheme uses an optimally determined ring thickness and hop size pair while SVHS and AVHS schemes vary hop size and its associated duty cycles over the entire lifetime. In SVHS, all sensors use same hop size concomitantly but it is ring-wise different in AVHS scheme. Performance analysis shows substantial improvement of network lifetime (above 130, 150, and 200 percent in FHS, SVHS, and AVHS, respectively, for path loss factor $\gamma=4$ and above 200, 250, and 300 percent, respectively, for $\gamma=2$ ) over the existing single hop, multihop or their hybrid transmission policies irrespective of network size and path loss factor. Energy usage is more uniformly distributed over the rings and critical energy per data cycle is reduced significantly in our policies. Moreover, we presented heuristics for distributed implementation of the proposed transmission policies.

The proposed policies are usable under node mobility as well. When a node moves to a new location, it can recalculate the optimal transmission parameters that will maximize its lifetime based on residual energy and new location. Appropriate regulation of hop sizes and their associated duty cycles based on sensors location can also be utilized to meet the delay requirements for time critical applications. Future work will focus on formulating a delay

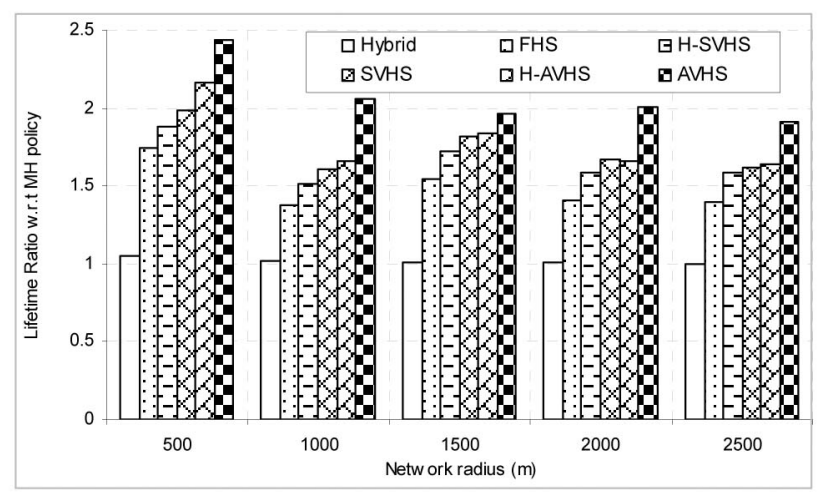

Fig. 7. The ratio of network lifetime achievable in FHS, H-SVHS, SVHS, $\mathrm{H}-\mathrm{AVHS}$, and AVHS transmissions to that of $\mathrm{MH}$ transmission for varying network radius $(\gamma=4)$.

and mobility aware transmission policy for clustered heterogeneous sensor networks.

\section{ApPENDix A}

1. Let $\Delta E_{k}$ be the difference of energy consumption by a sensor per data cycle in ring $C_{k}+1$ and $C_{k}, 1 \leq k<\eta$, hence

$$
\begin{aligned}
\Delta E_{k} & =e_{s}(k+1, w, \eta)-e_{s}(k, w, \eta) \\
& =\frac{\beta w^{\gamma}\left(l^{2}+\eta l-l\right)}{\eta(2 k-1)(2 k+1)}\left[-\frac{4 \alpha}{\beta w^{\gamma}}+\mho_{k}\right] \lambda_{s},
\end{aligned}
$$

where $\mho_{k}=(2 k-1)(k+1)^{\gamma}-(2 k+1) k^{\gamma}$ is always positive and an increasing function of $k$. Thus, $\Delta E_{k}$ is an increasing function of $k$ and its value may be positive or negative depending on the parameters like $\alpha, \beta, \gamma, w, \eta$, and $j$. We consider following three cases:

Case I $\Delta E_{k}>0, \forall k$. Energy consumption per data cycle by a sensor increases from ring $C_{1}$ to $C_{\eta}$.

Case II $\Delta E_{k}<0, \forall k$. Energy consumption per data cycle by a sensor decreases from ring $C_{1}$ to $C_{\eta}$.

Case III $\left(\Delta E_{1}, \ldots, \Delta E_{k-1}\right)<0$ and $\left(\Delta E_{k}, \ldots\right.$, $\left.\Delta E_{\eta-1}\right)>0$. There exists a $k$ between 2 to $\eta-1$ that switches the value of $\Delta E_{k}$ from negative to positive and remains positive for subsequent values. This gives the following relation:

$$
\begin{aligned}
& e_{s}(1, w, \eta)>e_{s}(2, w, \eta)>\cdots>e_{s}(k, w, \eta), \\
& e_{s}(k, w, \eta)<e_{s}(k+1, w, \eta)<\cdots<e_{s}(\eta, w, \eta) .
\end{aligned}
$$

Considering all three cases we can conclude that a sensor belonging ring $C_{1}$ or $C_{\eta}$ has the highest rate of energy consumption per data cycle and thus the critical ring is either $C_{1}$ or $C_{\eta}$ for any given ring thickness $w$ and hop size $\eta$. The energy consumption by a sensor in ring $C_{1}$ and $C_{\eta}$ per data cycle are $e_{s}(1, w, \eta)$ and $e_{s}(\eta, w, \eta)$, respectively. Using (14) to (16), ring $C_{1}$ is the critical ring if

$$
\begin{aligned}
& e_{s}(1, w, \eta) \geq e_{s}(\eta, w, \eta), \\
& \Rightarrow \frac{\eta^{\gamma}-2 \eta+1}{\eta-1} \leq \frac{4 \alpha}{\beta w^{\gamma}} .
\end{aligned}
$$

It is obvious that, in case of equality, the values for $e_{s}(1, w, \eta)$ and $e_{s}(\eta, w, \eta)$ will be same. 
2. For $\frac{\eta^{\gamma}-2 \eta+1}{\eta-1}=\frac{4 \alpha}{\beta w^{\gamma}}$, we get

$$
\Delta E_{k}=\frac{\beta w^{\gamma}\left(l^{2}+\eta l-l\right)}{\eta(2 k-1)(2 k+1)}\left[-\frac{\eta^{\gamma}-2 \eta+1}{\eta-1}+\mho_{k}\right] \lambda_{s}
$$

3. With $\eta>2$ and $2 \leq \gamma \leq 4$, simple calculation shows that, $\Delta E_{1}<0$ and $\Delta E_{\eta}>0$. This confirms the analysis in Case III that there always exists a ring whose energy consumption is minimum among rings $C_{1}$ to $C_{\eta}$. To find which ring's sensor has minimum per sensor energy usage per data cycle, we set the first order differentiation of (16) w.r.t. $i$ to zero that gives

$$
\frac{d}{d i} e_{s}(i, w, \eta)=(\gamma-1) i^{\gamma}-\frac{\gamma}{2} i^{\gamma-1}-\frac{2 \alpha}{\beta w^{\gamma}}=0
$$

\section{APPENDIX B}

From (14) and (15), for a given ring thickness $w$, the effect of changing hop size from $\eta$ to $\eta+1$ on the relay traffic and transmission range can be summarized as follows:

\begin{tabular}{|l|l|}
\hline Case I: $i \leq \eta$ & $I_{s}(i, w, \eta+1)<I_{s}(i, w, \eta)$ \\
& $x(i, w, \eta+1)=x(i, w, \eta)$ \\
\hline Case II: $\eta<i \leq l-\eta$ & $I_{s}(i, w, \eta+1)<I_{s}(i, w, \eta)$ \\
& $x(i, w, \eta+1)>x(i, w, \eta)$ \\
\hline Case III: $i>l-\eta$ & $I_{s}(i, w, \eta+1)=I_{s}(i, w, \eta)=0$ \\
& $x(i, w, \eta+1)>x(i, w, \eta)$ \\
\hline
\end{tabular}

From (16), we get $e_{s}(i, w, \eta)>e_{s}(i, w, \eta+1)$ and $e_{s}(i, w$, $\eta)<e_{s}(i, w, \eta+1)$ for Case I and Case III, respectively. But for Case II, there are two opposite events on the energy consumption due to changing hop size from $\eta$ and $\eta+1$ : 1) increase in transmission distance pushes toward more energy consumption, and 2) reduction in relay traffic leads to less energy consumption. However, the exponential term $\beta\{x(i, w, \eta+1)\}^{\gamma}$ in (16) becomes the dominating factor over $I_{s}(i, w, \eta+1)$ for typical energy and network parameters, and the overall energy consumption increases with the increase of hop size; hence $e_{s}(i, w, \eta+1)>e_{s}(i, w, \eta)$.

\section{APPENDIX C}

In this section, we solve the SVHS optimization problem using KKT conditions where the corresponding functions are given from (29) to (31). From (29) and (30) we get following constraints for $1 \leq i \leq \hat{l}$ :

$$
\begin{aligned}
Q_{i}(\boldsymbol{\Phi}) & =\Phi(1) B(i, 1)+\cdots+\Phi(\hat{l}) B(i, \hat{l})-E_{\text {init }} \leq 0, \\
\Phi(i) & \geq 0 .
\end{aligned}
$$

The solution of the optimization problem in Section 4.4 can be obtained from the following KKT conditions:

$$
\begin{aligned}
\sum_{i=1}^{\hat{l}} \mu_{i} \nabla Q_{i}(\boldsymbol{\Phi})+\sum_{i=1}^{\hat{l}} \sigma_{i} \nabla(-\Phi(i))-\nabla\|\boldsymbol{\Phi}\|_{1} & =0, \\
\mu_{i} Q_{i}(\boldsymbol{\Phi}) & =0, \\
\sigma_{i} \Phi(i) & =0,
\end{aligned}
$$

where $\mu_{i} \geq 0, \sigma_{i} \geq 0$ and the gradient vectors are given by:

$$
\begin{aligned}
& \nabla\|\boldsymbol{\Phi}\|_{1}=\left[\begin{array}{lll}
1 & \cdots & 1
\end{array}\right]^{\mathrm{T}}, \\
& \nabla Q_{i}(\boldsymbol{\Phi})=\left[\begin{array}{llll}
B(i, 1) & B(i, 2) & \cdots & B(i, \hat{l})
\end{array}\right]^{\mathrm{T}}, \\
& \nabla(-\Phi(i))=\left[\begin{array}{lllll}
0 & \cdots & -1 & \cdots & 0
\end{array}\right]^{\mathrm{T}} . \\
& i \text { th }{ }^{\uparrow} \text { row }
\end{aligned}
$$

Substituting the gradient vectors in the KKT conditions yields, for $1 \leq i \leq \hat{l}$

$$
\begin{aligned}
\sum_{j=1}^{\hat{l}} \mu_{j} B(j, i)-\sigma_{i}-1 & =0, \\
\mu_{i}\left[\sum_{j=1}^{\hat{l}} \Phi(j) B(i, j)-E_{\text {init }}\right] & =0, \\
-\sigma_{i} \Phi(i) & =0 .
\end{aligned}
$$

From (c1) and (c3) we get,

$$
\Phi(i)\left[\sum_{j=1}^{\hat{l}} \mu_{j} B(j, i)-1\right]=0 .
$$

We relax our original optimization problem by assuming that duty cycle for each hop size be at least greater than zero, i.e., $\Phi(i)>0,1 \leq i \leq \hat{l}$. This gives a system of linear equations from (c1) and can be expressed as

$$
\mu \mathrm{B}=1,
$$

where $\mu=\left[\mu_{1}, \mu_{2}, \ldots, \mu_{\hat{l}}\right]$ and $\mathbf{B}$ is given in (27). Decomposing $\mathbf{B}$ into a lower triangular matrix $\mathbf{L}=[L(i, j)]_{\hat{i} \times \hat{l}}$, and a upper triangular matrix $\mathbf{U}=[U(i, j)]_{\hat{l} \times \hat{l}}$, using crout's technique, gives

$$
\mu \mathbf{L} \mathbf{U}=1,
$$

where

$$
\begin{aligned}
& L(i, j)=B(i, j)-\sum_{k=1}^{j-1} L(i, k) U(k, j), \quad i \geq j, \text { and } \\
& U(i, j)= \begin{cases}1, & i=j, \\
\frac{1}{L(i, i)}\left[B(i, j)-\sum_{k=1}^{i-1} L(i, k) U(k, j)\right], & i<j .\end{cases}
\end{aligned}
$$

Let $\mathbf{Y}=\mu \mathbf{L}$, which gives $\mathbf{Y U}=\mathbf{1}$. Solving these two systems we get:

$$
\begin{aligned}
y_{i} & =1-\sum_{j=1}^{i-1} U(j, i) y_{j}, \quad 1 \leq i \leq \hat{l}, \\
\mu_{i} & =\frac{1}{L(i, i)}\left[y_{i}-\sum_{j=i+1}^{\hat{l}} \mu_{j} L(j, i)\right], \quad 1 \leq i \leq \hat{l} .
\end{aligned}
$$

From (c6), we find through induction that $\mu_{i}$ s are positive for $1 \leq i \leq \hat{l}$. Then from KKT condition in (c2)

$$
\mathbf{B} \boldsymbol{\Phi}=\mathbf{I} E_{\text {init }} \Rightarrow \mathbf{L U \Phi}=\mathbf{I} E_{\text {init }} .
$$

Let $\mathbf{Z}=\mathbf{U} \Phi$, which gives $\mathbf{L Z}=\mathbf{I} E_{\text {init }}$. Solving these systems gives: 


$$
\begin{gathered}
z_{i}=\frac{1}{L(i, i)}\left[E_{\text {init }}-\sum_{j=1}^{i-1} z_{j} L(i, j)\right], \quad 1 \leq i \leq \hat{l}, \\
\Phi(i)=z_{i}-\sum_{j=i+1}^{\hat{l}} \Phi(j) U(i, j), \quad 1 \leq i \leq \hat{l} .
\end{gathered}
$$

\section{REFERENCES}

[1] G. Pottie and W. Kaiser, "Wireless Integrated Network Sensors," Comm. of the ACM, vol. 43, no, 5, pp. 51-58, 2000.

[2] I.F. Akyildiz, W. Su, Y. Sankarsubramaniam, and E. Cayirci, "Wireless Sensor Networks: A Survey," Computer Networks, vol. 38, no. 4, pp. 393-422, 2002.

[3] S. Aeron, V. Saligrama, and D.A. Castanon, "Efficient Sensor Management Policies for Distributed Target Tracking in Multihop Sensor Networks," IEEE Trans. Signal Processing, vol. 56, no. 6, pp. 2562-2574, June 2008.

[4] H. Liu, P. Wan, and X. Jia, "Maximal Lifetime Scheduling for Sensor Surveillance Systems with K Sensors to One Target," IEEE Trans. Parallel and Distributed Systems, vol. 15, no. 2, pp. 334-345, Apr. 2007.

[5] W.R. Heinzelman, A. Chandrakasan, and H. Balakrishnan, "An Application-Specific Protocol Architecture for Wireless Microsensor Networks," IEEE Trans. Wireless Comm., vol. 1, no. 4, pp. 660670, Oct. 2002.

[6] Y.C. Tseng, M.S. Pan, and Y.Y. Tsai, "Wireless Sensor Networks for Emergency Navigation," IEEE Computer, vol. 39, no. 7, pp. 5562, July 2006.

[7] K. Akkaya and M. Younis, "A Survey on Routing for Wireless Sensor Networks," Ad Hoc Networks, vol. 3, no. 3, pp. 325-349, 2005.

[8] W. Ye, J. Hiedemann, and D. Estrin, "Medium Access Control with Coordinated Adaptive Sleeping for Wireless Sensor Networks," IEEE/ACM Trans. Networking, vol. 12, no. 3, pp. 493-506, June 2004.

[9] I. Demir, C. Ersoy, and F. Alagoz, "MAC Protocols for Wireless Sensor Networks: A Survey," IEEE Comm. Magazine, vol. 44, no. 4, pp. 115-121, Apr. 2006.

[10] I. Rhee, A. Warrier, M. Aia, J. Min, and M.L. Sichitiu, "Z-MAC: A Hybrid MAC for Wireless Sensor Networks," IEEE/ACM Trans. Networking, vol. 16, no. 3, pp. 511-524, June 2008.

[11] W. Choi and S.K. Das, "A Novel Framework for EnergyConserving Data Gathering in Wireless Sensor Networks," Proc. IEEE INFOCOM, pp. 1985-1996, Mar. 2005.

[12] O. Younis and S. Fahmy, "HEED: A Hybrid, Energy-Efficient, Distributed Clustering Approach for Ad Hoc Sensor Networks," IEEE Trans. Mobile Computing, vol. 3, no. 4, pp. 366-379, Oct.-Dec. 2004.

[13] M. Bhardwaj, T. Garnett, and A.P. Chandrakasan, "Upper Bounds on Lifetime of Sensor Networks," IEEE Int'l Conf. Comm. (ICC), pp. 785-790, 2001.

[14] V. Mhatre and C. Rosenberg, "Design Guidelines for Wireless Sensor Networks: Communication, Clustering and Aggregation," Ad Hoc Networks, vol. 2, no. 1, pp. 45-63, 2004.

[15] C. Efthymiou, S. Nikoletseas, and J. Rolim, "Energy Balanced Data Propagation in Wireless Sensor Networks," Wireless Networks, vol. 12, no. 6, pp. 691-707, 2006.

[16] V. Mhatre, C. Rosenberg, D. Kofman, R. Mazumder, and N. Shroff, "A Minimum Cost Heterogeneous Sensor Network with a Lifetime Constraint," IEEE Trans. Mobile Computing, vol. 4, no. 1, pp. 4-15, Jan./Feb. 2005.

[17] F. Sivrikaya and B. Yener, "Time Synchronization in Sensor Networks," IEEE Network, vol. 18, no. 4, pp. 45-50, July/Aug. 2004.

[18] S. Tilak, N.B. Abu-Ghazaleh, and W. Heinzelman, "A Taxonomy of Wireless Micro-Sensor Networks Models," ACM Mobile Computing and Comm. Rev., vol. 6, no. 2, pp. 28-36, 2002.

[19] P. Gupta and P.R. Kumar, "Critical Power for Asymptotic Connectivity in Wireless Networks," Analysis, Control, Optimization and Applications, pp. 547-566, 1998.

[20] R. Madan, S. Cui, S. Lal, and A.J. Goldsmith, "Modeling and Optimization of Transmission Schemes in Energy-Constrained Wireless Sensor Networks," IEEE/ACM Trans. Networking, vol. 15, no. 6, pp. 1359-1372, Dec. 2007.
[21] O. Powell, P. Leone, and J. Rolim, "Energy Optimal Data Propagation in Wireless Sensor Networks," J. Parallel and Distributed Computing, vol. 67, no. 3, pp. 302-317, 2007.

[22] P. Leone, S. Nikoletseas, and J. Rolim, "Stochastic Models and Adaptive Algorithms for Energy Balance in Sensor Networks," Theory of Computing Systems, vol. 47, no. 2, pp. 433-453, 2009.

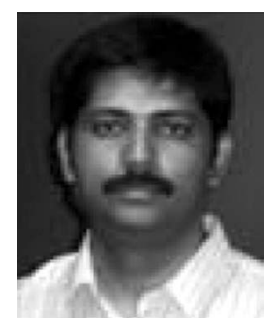

A.K.M. Azad received the $\mathrm{PhD}$ degree from the faculty of Information Technology, Monash University, Australia, in May 2010. He also received the BSc and MSc degrees from the Department of Computer Science and Engineering (CSE), Bangladesh University of Engineering and Technology (BUET), Dhaka, Bangladesh, in 1998 and 2005, respectively. Currently, Dr. Azad is working as a research engineer in the Department of Electrical and Computer Systems Engineering, Faculty of Engineering, Monash University, Australia. His research interests include wireless communications, especially, ad hoc and sensor networks, RFID sensor networks, smart antenna and RFID systems, signal processing, future Internet, algorithms, and graph theory. He is a student member of the IEEE.

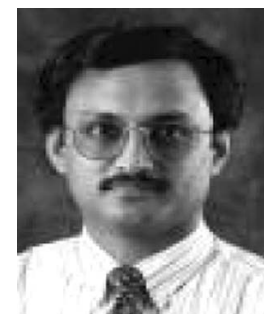

Joarder Kamruzzaman is currently a faculty member in the Faculty of Information Technology, Monash University, Australia. He has published more than 145 peer-reviewed publications which include 30 journal papers and five book chapters. He has edited two reference books and served as a program committee member of a number of international conferences. His research interests include sensor networks, ad hoc networks, and cognitive radios. $\mathrm{He}$ is a member of the IEEE.

$\triangleright$ For more information on this or any other computing topic, please visit our Digital Library at www.computer.org/publications/dlib. 